01,02

\title{
Частота и быстродействие спинового вентиля с планарной анизотропией слоев
}

\author{
() Ю.А. Юсипова \\ Институт проблем проектирования в микроэлектронике РАН, \\ Москва, Россия \\ E-mail: linda_nike@mail.ru \\ Поступила в Редакцию 26 марта 2020 г. \\ В окончательной редакции 26 марта 2020 г. \\ Принята к публикации 2 апреля 2020 г.
}

\begin{abstract}
Проведено моделирование динамики вектора намагниченности свободного слоя слоистой спин-вентильной структуры. В качестве материалов для свободного и закрепленного слоев рассмотрены шесть магнитомягких ферромагнетиков с планарной анизотропией. Выделены типы динамики намагниченности, представляющие практический интерес для MRAM и HMDD (переключение вектора намагниченности), STNO (устойчивая прецессия вектора намагниченности), и базового элемента PSL (переключение вектора намагниченности с двумя вероятными исходами). Рассчитаны диапазоны токов и полей, соответствующие этим режимам работы спинового вентиля. Проведенные численные расчеты времени переключения показали, что среди рассмотренных материалов наиболее подходящим для ячейки MRAM является сплав $\mathrm{Co}_{80} \mathrm{Gd}_{20}$, a для считывающей головки $\mathrm{HMDD}-\mathrm{Fe}_{60} \mathrm{Co}_{20} \mathrm{~B}_{20}$. В результате расчета частоты прецессии сделан вывод, что оптимальным для изготовления ферромагнитных слоев STNO является сплав $\mathrm{Fe}_{60} \mathrm{Co}_{20} \mathrm{~B}_{20}$. Для реализации PSL наилучшие переключательные характеристики продемонстрировал сплав $\mathrm{Co}_{93} \mathrm{Gd}_{7}$.
\end{abstract}

Ключевые слова: спиновый вентиль, магниторезистивная память с произвольным доступом, вероятностная спиновая логика, спин-трансферный наноосциллятор, считывающая головка жесткого диска.

DOI: 10.21883/FTT.2020.09.49754.29H

\section{1. Введение}

В классической теории магнитные состояния ферромагнетиков управляются приложенным магнитным полем. В 1996 г. Слончевский [1] предсказал другой способ изменения магнитной конфигурации наномагнитов с помощью спин-поляризованного тока. Угловой момент, переносимый спин-поляризованным током, передает крутящий момент вектору намагниченности, что приводит к его переключению в новое состояние равновесия или постоянной прецессии. Изменение проекции вектора намагниченности на ось анизотропии служит причиной изменения магнетосопротивления слоистой спин-вентильной структуры, что влечет за собой колебания напряжения во внешней цепи. Простейшая конфигурация такой структуры состоит из относительно толстого „закрепленного“ ферромагнитного слоя, который служит поляризатором тока, немагнитной прослойки и относительно тонкого „свободного“ слоя. Слой антиферромагнетика нужен для фиксации намагниченности закрепленного слоя. В качестве немагнитной прослойки используют немагнитный металл (например, медь) или тонкий диэлектрик (например, $\mathrm{MgO}$ ), соответствующие структуры обычно называют спиновым вентилем или магнитным туннельным переходом (MTJ).

Спин-вентильные структуры нашли применение в большом количестве микроэлектронных устройств это магниторезистивная память с произвольным доступом (MRAM) [2,3], считывающие головки жестких дисков (HMDD) [4], различные спин-трансферные наноосцилляторы (STNO) [5]. Они также используются для реализации детерминированной (SL) [6] и вероятностной спиновой логики (PSL) [7-12].

Магниторезистивная память MRAM - это универсальная память, которая способна выполнять функцию внутрисхемно-программируемой памяти, быстрого буфера и энергонезависимого хранилища данных. MRAM имеет множество преимуществ перед другими видами памяти, таких как энергонезависимость, бесконечное количество циклов перезаписи, широкий температурный диапазон, высокие быстродействие и радиационная стойкость [2,3]. Магниторезистивная память MRAM на основе ферробората кобальта, изготовленная корпорацией IBM в сотрудничестве с компанией Samsung в 2016 г., имеет время записи $10 \mathrm{~ns}$ при токе записи $7.5 \mu \mathrm{A}$ [4]. В теоретической работе [5] рассмотрены ячейки MRAM на основе ферромагнетиков $\mathrm{TbFeCo}$ и $\mathrm{GdFeCo}$ с различными немагнитными прослойками. Критический ток переключения для этих ячеек MRAM колеблется в диапазоне 1-20 mA, а критическое поле переключения в диапазоне $0.8-15.9 \mathrm{kA} / \mathrm{m}$. Время переключения при этих параметрах лежит в интервале $1-30 \mathrm{~ns}$.

Введение спин-вентильного сенсора в архитектуру магниторезистивных считывающих головок HMDD обеспечивает их высокую чувствительность, что способствует увеличению поверхностной плотности записи на три порядка. Темп роста емкости жесткого диска с их появлением возрос до 100\% в год [3]. 
Наноразмерные микроволновые осцилляторы STNO обладают возможностью изменения частоты осцилляций в широком диапазоне с помощью приложенного электрического тока и магнитного поля. Высокая степень интеграции и совместимость с К-МОП технологией дает им возможность конкурировать с используемыми в настоящее время генераторами микроволнового излучения [6]. Спин-трансферный наноосциллятор на основе ферробората кобальта, рассмотренный в работе [7], показал диапазон рабочих частот возбуждаемых осцилляций $1-10 \mathrm{MHz}$ для плотности тока $10^{6}-10^{8} \mathrm{~A} / \mathrm{cm}^{2}$. В то же время STNO на основе пермаллоя в поле $24 \mathrm{mT}$, в диапазоне токов 3-15 mA имеет частоту работы от 0.8 до $1.2 \mathrm{GHz}[8]$.

Базовым элементом вероятностной спиновой логики (PSL) является бинарный стохастический нейрон (p-bit). PSL лежит в основе технологии нейронных сетей, использующих байесовский статистический вывод [9] и обратимую булеву логику [10], в основе квантовых компьютеров на базе модели Изинга [11-14], а также устройств распознавания изображений [15] и генераторов случайных чисел. В работах [9-15] представлена модель стохастического нейрона на основе МТJ, переключение которого производиться посредствам спинового эффекта Холла. В этом случае расчеты были проведены для двух ферромагнетиков с энергетическим барьером 48 и $12 \mathrm{kBT}$. Ток переключения в данном случае составляет $1 \mathrm{~mA}$.

Основная проблема спин-вентильных микроэлектронных устройств - большие величины управляющих параметров (электрического тока и магнитного поля). Поэтому для улучшения энергоэффективности сравнение критических характеристик переключения и прецессии спинового вентиля на основе различных материалов представляет практический интерес.

Основной задача данной работы - расчет времени и вероятности переключения, а также частоты осцилляций спинового вентиля под действием спин-поляризованного тока и магнитных полей различного направления. Целью исследования является подбор наиболее подходящих ферромагнитных материалов и конфигурации магнитного поля, обеспечивающих наилучшие переключательные характеристики MRAM и HMDD, частотные характеристики STNO и быстродействие элементов PSL. В настоящей работе предложена новая модель стохастического нейрона p-bit на основе спинового вентиля с металлической немагнитной прослойкой, переключаемого спинполяризованным током в геометрии „ток перпендикулярен плоскости“. Данная модель предполагает большую степень интеграции и меньшую энергозатратность, чем модель, представленная в работах [9-15].

\section{2. Основные уравнения}

Объектом данного исследования является спиновый вентиль с планарной анизотропией слоев. Сторона квад-

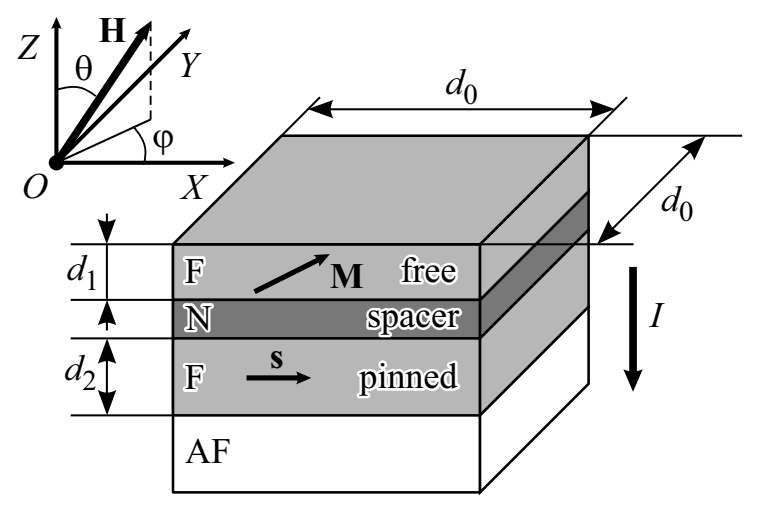

Рис. 1. Спиновый вентиль.

ратного поперечного сечения вентиля $d_{0}=11 \mathrm{~nm}$ [4]. Ось анизотропии направлена вдоль одной из сторон квадрата. Толщина свободного слоя $d_{1}=2 \mathrm{~nm}$, толщина закрепленного слоя $d_{2}=5 \mathrm{~nm}$, а толщина медной немагнитной прослойки $d_{N}=1.2 \mathrm{~nm}$ [16]. Ось $O X$ системы координат, связанной со структурой, направлена вдоль оси анизотропии. Структура помещена в магнитное поле $\mathbf{H}$, которое составляет углы $\varphi$ и $\theta$ с осями $O X$ и $O Z$. Электрический ток плотностью $J$ пропускается перпендикулярно плоскости слоев. Вектор М - это вектор намагниченности свободного слоя спинового вентиля, a $M_{X}, M_{Y}, M_{Z}$ - его проекции на оси $O X, O Y, O Z$ соответственно. Направление единичного вектора s совпадает с направлением намагниченности закрепленного слоя (рис. 1).

В качестве материалов для ферромагнитных слоев рассмотрены кобальт Со и железо $\mathrm{Fe}$ (монокристаллические пленки которых легче и дешевле получить), сплавы $\mathrm{Fe}_{60} \mathrm{Co}_{20} \mathrm{~B}_{20}$ и $\mathrm{Fe}_{70} \mathrm{Co}_{30}$ (имеющие высокий параметр спиновой поляризации $P$ ), а также $\mathrm{Co}_{93} \mathrm{Gd}_{7}$ и $\mathrm{Co}_{80} \mathrm{Gd}_{20}$, имеющие невысокие коэффициенты анизотропии. Намагниченность насыщения $M_{s}$, коэффициент диссипации $\alpha$, константа анизотропии $K$ и параметр спиновой поляризации $P$ представлены в табл. 1. Дефекты микроструктуры материалов в нашей модели не учитывались.

Динамика вектора намагниченности свободного слоя спинового вентиля М описывается уравнением: Ландау-

Таблица 1. Магнитные параметры различных магнитомягких материалов [17]

\begin{tabular}{l|c|c|r|c}
\hline Материал & $\mu_{0} M_{s}, \mathrm{~T}$ & $\alpha$ & $K, \mathrm{~J} / \mathrm{m}^{3}$ & $P$ \\
\hline $\mathrm{Co}$ & 0.1 & 0.02 & 1380 & 0.35 \\
$\mathrm{Fe}$ & 1.21 & 0.02 & 1880 & 0.40 \\
$\mathrm{Fe}_{70} \mathrm{Co}_{30}$ & 1.76 & 0.02 & 530000 & 0.55 \\
$\mathrm{Fe}_{60} \mathrm{Co}_{20} \mathrm{~B}_{20}$ & 1.96 & 0.04 & 210000 & 0.52 \\
$\mathrm{Co}_{93} \mathrm{Gd}_{7}$ & 2.15 & 0.008 & 48000 & 0.30 \\
$\mathrm{Co}_{80} \mathrm{Gd}_{20}$ & 2.4 & 0.015 & 35000 & 0.1
\end{tabular}


Таблица 2. Параметры спинового вентиля для различных материалов ферромагнитных слоев

\begin{tabular}{l|c|r|r|c}
\hline Материал & $\rho, 10^{-8} \Omega \cdot \mathrm{m}$ & $R_{A P}, \Omega$ & $R_{P}, \Omega$ & $G M R, \%$ \\
\hline $\mathrm{Co}$ & $6.24[22]$ & 4.11 & 3.70 & 11 \\
$\mathrm{Fe}$ & $9.71[22]$ & 6.58 & 5.71 & 15 \\
$\mathrm{Fe}_{70} \mathrm{Co}_{30}$ & 8.33 & 7.62 & 4.93 & 34 \\
$\mathrm{Fe}_{60} \mathrm{Co}_{20} \mathrm{~B}_{20}$ & $19.56[24]$ & 15.01 & 11.42 & 31 \\
$\mathrm{Co}_{93} \mathrm{Gd}_{7}$ & 7.81 & 4.97 & 4.61 & 8 \\
$\mathrm{Co}_{80} \mathrm{Gd}_{20}$ & 11.29 & 6.67 & 6.62 & 1
\end{tabular}

Лифшица-Гильберта

$$
\begin{aligned}
\frac{\partial \mathbf{M}}{\partial t}= & -|\gamma| \mu_{0}\left[\mathbf{M} \times \mathbf{H}_{\mathrm{eff}}\right] \\
& -\gamma G \frac{J \hbar}{e d_{1}}[\mathbf{M} \times[\mathbf{s} \times \mathbf{M}]]+\frac{\alpha}{M_{s}}\left[\mathbf{M} \times \frac{\partial \mathbf{M}}{\partial t}\right],
\end{aligned}
$$

где $\gamma$ - гиромагнитное отношение, $G$ - токовый член в форме Слончевского-Берже, который можно выразить следующим уравнением

$$
G=4 P^{3 / 2}\left((1+P)^{3}\left(3+\left(\mathbf{s}, \frac{\mathbf{M}}{M_{s}}\right)\right)-16 P^{3 / 2}\right)^{-1} .
$$

Эффективное магнитное поле $\mathbf{H}_{\mathrm{eff}}$ включает:

- поле магнитной анизотропии $2 M_{X} K /\left(M_{s}^{3} \mu_{0}\right)$, направленное вдоль оси $O X$;

- поле размагничивания $M_{Z}$, направленное вдоль оси $O Z$;

- внешнее магнитное поле $\mathbf{H}$.

Детали бифуркационного анализа динамической системы (1) приведены в работах [2,17-19].

Изменение проекции вектора намагниченности М на ось анизотропии $O X$ из-за эффекта гигантского магнетосопротивления приводит к изменению выходного сигнала $U$ [20]. Его величина определяется следующим выражением

$$
U=J d_{0}^{2}\left(\frac{R_{p}+R_{A P}}{2}+\frac{R_{p}-R_{A P}}{2} \frac{M_{X}}{M_{s}}\right),
$$

где $R_{P}$ и $R_{A P}$ - сопротивления спинового вентиля в параллельном и антипараллельном состояниях (табл. 2) соответственно. Согласно резисторной модели гигантского магнетосопротивления для структуры в выбранной геометрии „ток перпендикулярен плоскости“ выражения для сопротивления $R_{P}$ и $R_{A P}$ можно представить в виде [21]:

$$
\begin{gathered}
R_{P}=\frac{\left(\left(d_{2}+d_{1}\right) \rho_{u p}+d_{N} \rho_{N}\right)\left(\left(d_{2}+d_{1}\right) \rho_{\text {down }}+d_{N} \rho_{N}\right)}{d_{0}^{2}\left(\left(d_{2}+d_{1}\right) \rho_{u p}+2 d_{N} \rho_{N}+\left(d_{2}+d_{1}\right) \rho_{\text {down }}\right)}, \\
R_{A P}=\frac{\left(d_{2} \rho_{\text {down }}+d_{1} \rho_{\text {up }}+d_{N} \rho_{N}\right)\left(d_{2} \rho_{\text {up }}+d_{1} \rho_{\text {down }}+d_{N} \rho_{N}\right)}{d_{0}^{2}\left(\left(d_{2}+d_{1}\right) \rho_{u p}+2 d_{N} \rho_{N}+\left(d_{2}+d_{1}\right) \rho_{\text {down }}\right)},
\end{gathered}
$$

где $\rho_{\text {up, down }}=2 \rho / 1 \pm P, \rho-$ удельное сопротивление ферромагнитных слоев (табл. 2), $\rho_{N}$ - удельное сопротивление немагнитной прослойки (для меди
$\left.\rho_{N}=1.67 \cdot 10^{-8} \Omega \cdot \mathrm{m}[22]\right)$. Методика расчета удельного сопротивления двухкомпонентных сплавов подробно описана в работе [23]. Однако для практических применений удельное сопротивление используемых пленок должно быть измерено экспериментально. В табл. 2 представлены коэффициенты гигантского магнетосопротивления $G M R=\left(R_{A P}-R_{P}\right) / R_{P}$ для спиновых вентилей на основе различных материалов.

\section{3. Динамика вектора намагниченности}

Динамика вектора М была рассчитана путем решения уравнения (1) методом Рунге-Кутты, при этом возмущение относительно положения равновесия было принято равным 0.0001 .

Основные типы динамики, имеющие практическую ценность для PSL и STNO - это переключательный режим с двумя вероятными исходами и устойчивая прецессионная мода, соответственно. В то время как базовым режимом работы MRAM и HMDD является переключение из параллельного состояния в антипараллельное и наоборот. Режим прецессии с точки зрения качественной теории динамических систем представляет собой предельный цикл.

\section{1. Переключение вектора намагниченности}

Процесс записи логической „1“ в ячейку MRAM соответствует смене направления вектора намагниченности свободного слоя М от параллельного на антипараллельное направлению вектора намагниченности закрепленного слоя s. Параллельное направление вектора М эквивалентно точке равновесия $T_{1}$, а антипараллельное - положению равновесия $T_{2}$ (рис. 2). Переключение спинового вентиля происходит при воздействии электрического тока. Записи логической „1“ соответствует направление тока, противоположное оси $O Z$, а записи логического „0“ - совпадающее с осью $O Z$.

B работе HMDD используется переключательный режим, при котором изменение направления вектора М происходит под действием магнитного поля ячейки памяти HMDD. Для переключения вектора $\mathbf{M}$ из точки $T_{1}$ в точку $T_{2}$ необходимо приложить магнитное поле, антипараллельное оси $O X(\varphi=\pi, \theta=\pi / 2)$, а для переключения из положения равновесия $T_{2}$ в положение $T_{1}-$ параллельное оси $O X(\varphi=0, \theta=\pi / 2)$.

Рис. 2, $а$ демонстрирует две траектории переключения вектора М из точки $T_{1}$ в точку $T_{2}$, с отклонениями противоположных знаков, для спинового вентиля на основе $\mathrm{Fe}_{60} \mathrm{Co}_{20} \mathrm{~B}_{20}$ при $H=0$ и $J=1.30 \cdot 10^{8} \mathrm{~A} / \mathrm{cm}^{2}$. На рис. $2, b$ показана соответствующая вольт-секундная характеристика, полученная по формуле (2). Время переключения спинового вентиля $t_{1-2}$ в этом случае будет составлять 73 ns.

На рис. 3, $а$ представлен результат численного расчета обратной величины времени записи $t_{1-2}$ от плотности 


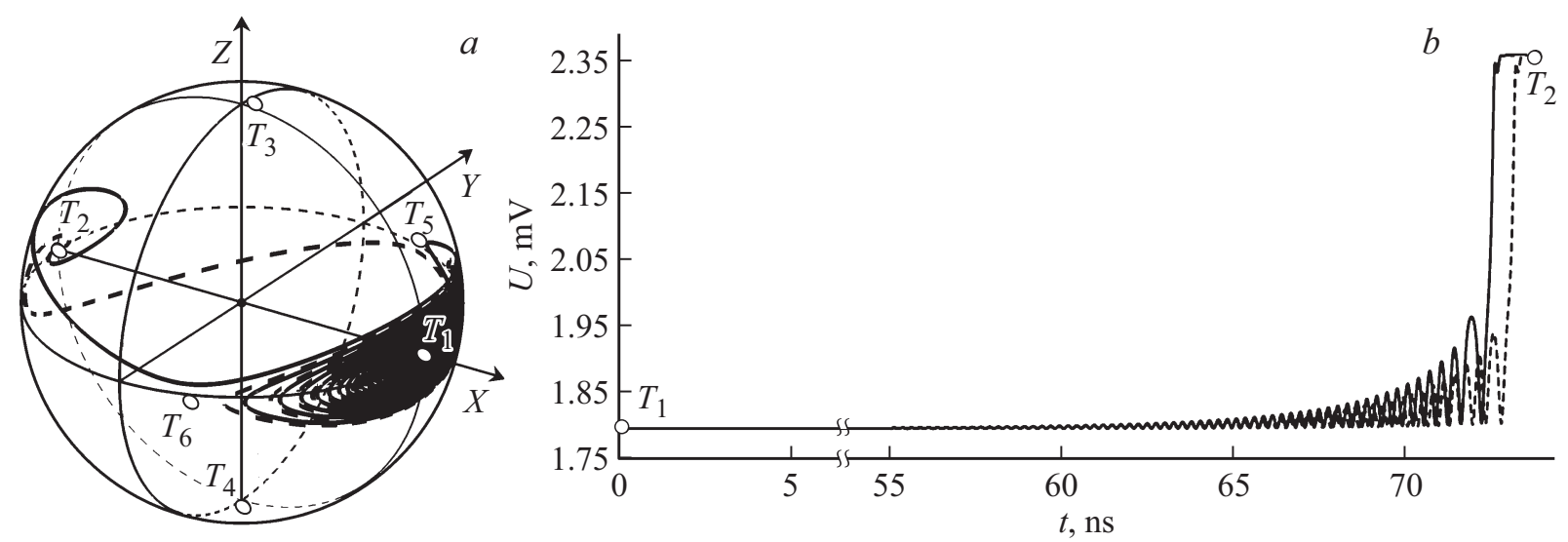

Рис. 2. Переключение намагниченности свободного слоя спинового вентиля на основе $\mathrm{Fe}_{60} \mathrm{Co}_{20} \mathrm{~B}_{20}(a)$; зависимость $U(t)$ при тех же параметрах $(b)$.
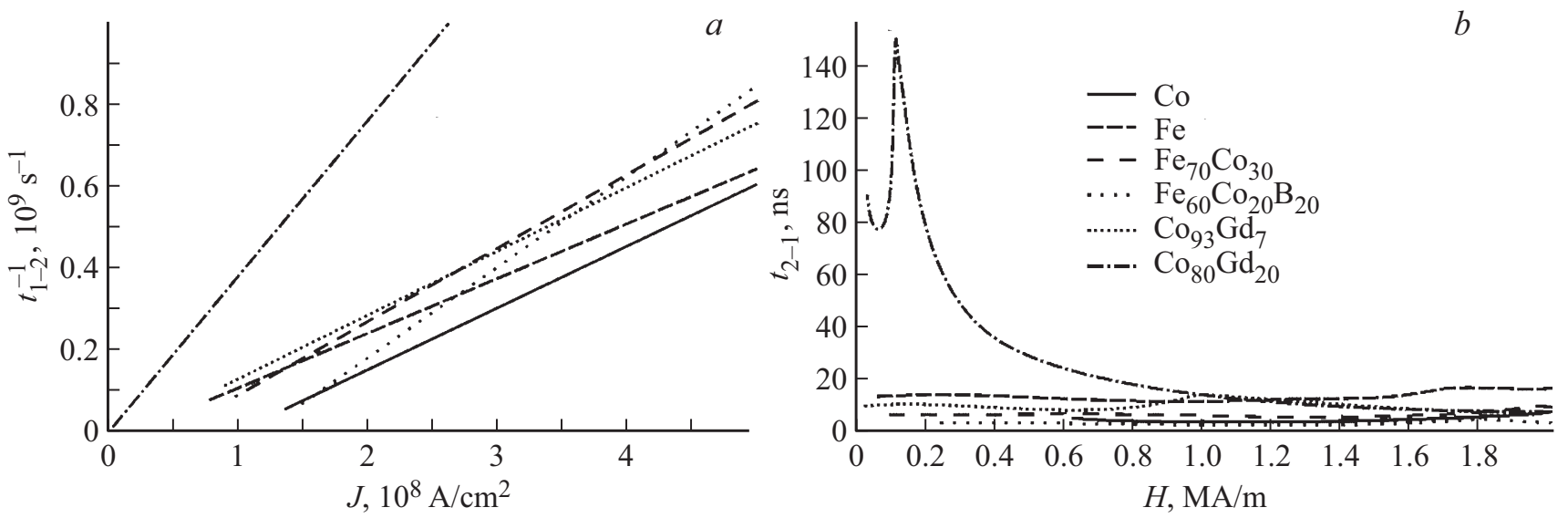

Рис. 3. Зависимость времени переключения спинового вентиля $t_{1-2}$ от плотности тока $(a)$; зависимость времени переключения $t_{2-1}$ от магнитного поля $H$ направленного вдоль оси $O X(b)$.

тока $J$ для различных материалов. Зависимость $t_{1-2}^{-1}(J)$ имеет линейный характер. Функция времени переключения $t_{2-1}$ вектора $\mathbf{M}$ из положения $T_{2}$ в положение $T_{1}$ от величины магнитного поля $H$ при $\varphi=0, \theta=\pi / 2$ показана на рис. $3, b$. Точка максимума $t_{2-1}(H)$ соответствует моменту изменения типа особой точки с седла на неустойчивый фокус. Зависимости $t_{1-2}^{-1}(J)$ и $t_{2-1}(H)$ численно рассчитаны с помощью моделирования динамики вектора М методом Рунге-Кутты и последующего анализа полученных годографов.

Спиновый вентиль на основе $\mathrm{Co}_{80} \mathrm{Gd}_{20}$ обладает наименьшим критическим током переключения $J_{\min }=1.56 \cdot 10^{6} \mathrm{~A} / \mathrm{cm}^{2}$, а спиновый вентиль на основе $\mathrm{Fe}_{60} \mathrm{Co}_{20} \mathrm{~B}_{20}$ - наибольшим $J_{\min }=1.25 \cdot 10^{8} \mathrm{~A} / \mathrm{cm}^{2}$. Вpeмя переключения спинового вентиля на основе $\mathrm{Co}_{80} \mathrm{Gd}_{20}$ при токе, близком к $J_{\min }$, составляет $960 \mathrm{~ns}$, однако при увеличении тока оно существенно снижается. При токе $J_{\min }=1.25 \cdot 10^{8} \mathrm{~A} / \mathrm{cm}^{2}$ время переключения для $\mathrm{Co}_{80} \mathrm{Gd}_{20}$ составляет всего $1.8 \mathrm{~ns}$, что в 9 раз меньше, чем время переключения для $\mathrm{Fe}_{60} \mathrm{Co}_{20} \mathrm{~B}_{20}$ при том же токе.
Наименьшим критическим магнитным полем переключения $H_{\min }=3.13 \cdot 10^{3} \mathrm{~A} / \mathrm{m}$ обладает спиновый вентиль, ферромагнитные слои которого изготовлены из $\mathrm{Co}_{93} \mathrm{Gd}_{7}$. Наименьшее время переключения $t_{2-1}$, равное 2-4ns, показал спиновый вентиль на основе $\mathrm{Fe}_{60} \mathrm{Co}_{20} \mathrm{~B}_{20}$. Время переключения $t_{2-1}$ для $\mathrm{Co}_{93} \mathrm{Gd}_{7}$ всегда в 3-5 раз больше, чем для $\mathrm{Fe}_{60} \mathrm{Co}_{20} \mathrm{~B}_{20}$, при равных значениях магнитного поля.

\section{2. Прецессия вектора намагниченности}

Для STNO важны те режимы с прецессией, в которых проекция вектора намагниченности на ось анизотропии периодически существенно изменяется, что приводит к значительному изменению выходного сигнала $U$ в течении периода.

На рис. 4, $a$ изображена динамика вектора М намагниченности свободного слоя спинового вентиля на основе $\mathrm{Co}_{80} \mathrm{Gd}_{20}$ в магнитном поле, параллельном оси $O X \quad\left(\varphi=0, \quad \theta=\pi / 2, \quad H=1.60 \cdot 10^{4} \mathrm{~A} / \mathrm{m}\right.$, $\left.J=1.67 \cdot 10^{7} \mathrm{~A} / \mathrm{cm}^{2}\right)$. При этом частота осцилляций $v$ 

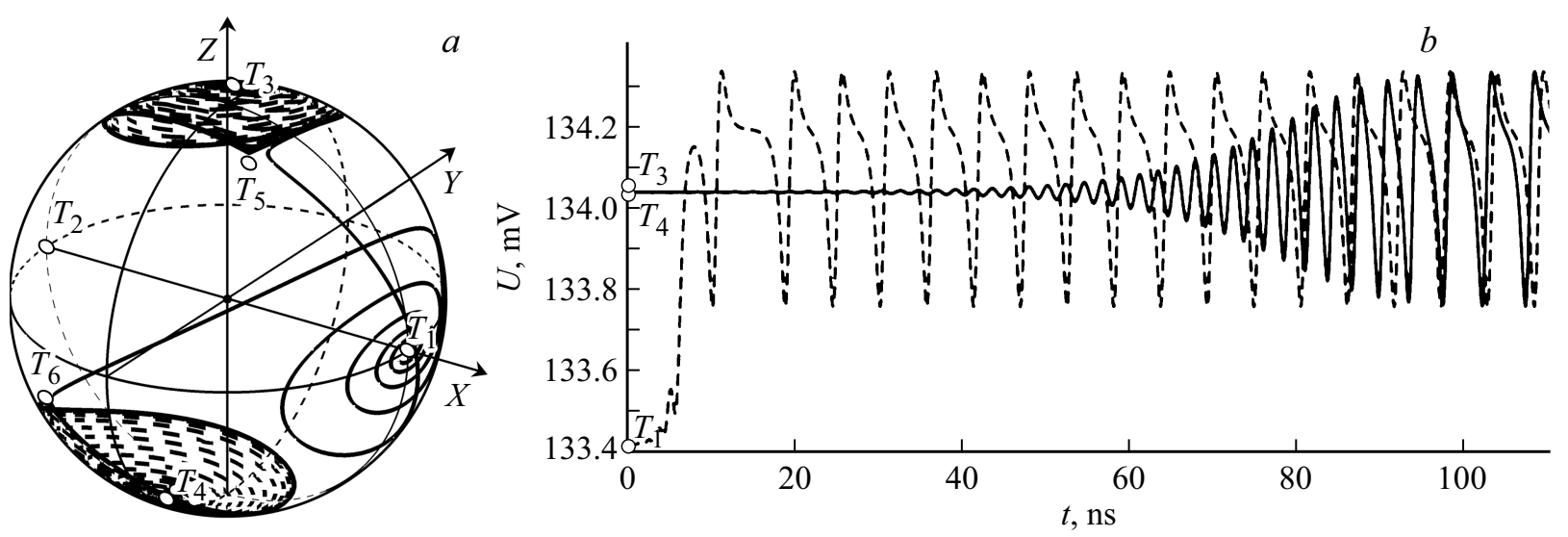

Рис. 4. Динамика вектора намагниченности свободного слоя спинового вентиля на основе $\mathrm{Co}_{80} \mathrm{Gd}_{20}$ в магнитном поле параллельном оси $O X(a)$; вольт-секундные характеристики при тех же параметрах $(b)$.
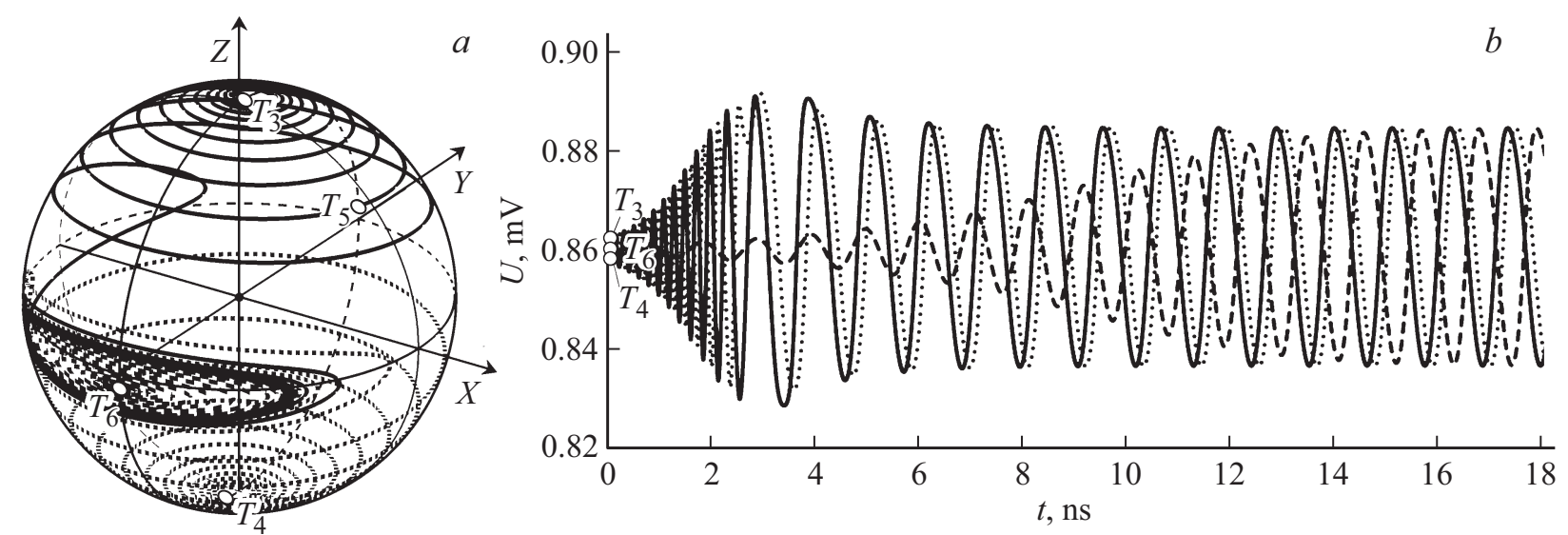

Рис. 5. Устойчивая прецессия вектора М спинового вентиля на основе $\mathrm{Co}_{93} \mathrm{Gd}_{7}$ в магнитном поле антипараллельном оси $O Y$ (a); временная зависимость выходного сигнала при тех же параметрах $(b)$.

составляет $0.18 \mathrm{GHz}$, а амплитуда колебаний $U_{\max }=0.35 \mu \mathrm{V}$.

Пары траекторий, вышедшие из точки $T_{1}, T_{3}$ и $T_{4}$, a также $T_{5}$ и $T_{6}$, симметричны относительно оси $O X$ (рис. $4, a$ ), поэтому имеют одинаковые вольт-секундные характеристики (рис. $4, b$ ). Вокруг неустойчивых фокусов $T_{3}$ и $T_{4}$ возникают два симметричных относительно оси $O X$ устойчивых предельных цикла. Время установления колебаний $\tau$ зависит от величины действительной части $R e_{T}$ собственных чисел матрицы линеаризации для точки, из которой исходит траектория. При данных условиях минимальным $R e_{T}=0.003$ обладают собственные числа в точках $T_{3}$ и $T_{4}-$ следовательно, для траекторий, выходящих из этих точек, характерно максимальное время установления колебаний $\tau_{\max }=97 \mathrm{~ns}$.

На рис. 5, $a$ представлена устойчивая прецессия для слоистой структуры с ферромагнитными слоями, изготовленными из $\mathrm{Co}_{93} \mathrm{Gd}_{7}$, который помещен в магнитное поле антипараллельное оси $O Y(\varphi=-\pi / 2, \theta=\pi / 2$, $\left.H=3.70 \cdot 10^{4} \mathrm{~A} / \mathrm{m}, J=1.48 \cdot 10^{8} \mathrm{~A} / \mathrm{cm}^{2}\right)$. Зависимость напряжения выходного сигнала $U$ от времени (рис. $5, b$ ) показывает, что максимальным временем установления колебаний $\tau_{\max }=17 \mathrm{~ns}$ обладает траектория конца вектора $\mathbf{M}$, вышедшая из точки $T_{6}$. Частота осцилляций $v$, в данном случае, составляет $0.6 \mathrm{GHz}$, а амплитуда колебаний $U_{\max }=24 \mu \mathrm{V}$.

На рис. 6, $a$ показана динамика вектора намагниченности свободного слоя спинового вентиля на основе железа в магнитном поле, параллельном оси $O Z(\varphi=0$, $\left.\theta=0, H=8.60 \cdot 10^{5} \mathrm{~A} / \mathrm{m}\right)$ при токе $J=2.76 \cdot 10^{8} \mathrm{~A} / \mathrm{cm}^{2}$, a на рис. $6, b$ соответствующая ему вольт-секундная характеристика. В этом случае частота и амплитуда осцилляций составляют $7.5 \mathrm{GHz}$ и $143 \mu \mathrm{V}$ соответственно. Максимальное время установления колебаний $\tau_{\max }$ характерно для траектории, выходящей из точки $T_{6}$, и составляет $10.8 \mathrm{~ns}$.

Следует отметить, что в случае, когда магнитное поле направлено параллельно оси $O Y$ или $O Z$, траектория конца вектора намагниченности М наматывается на предельный цикл вокруг оси, вдоль которой направлено магнитное поле, а вольт-секундные характеристики приближаются к гармоническим (рис. $5, b, 6, b$ ). В то же 


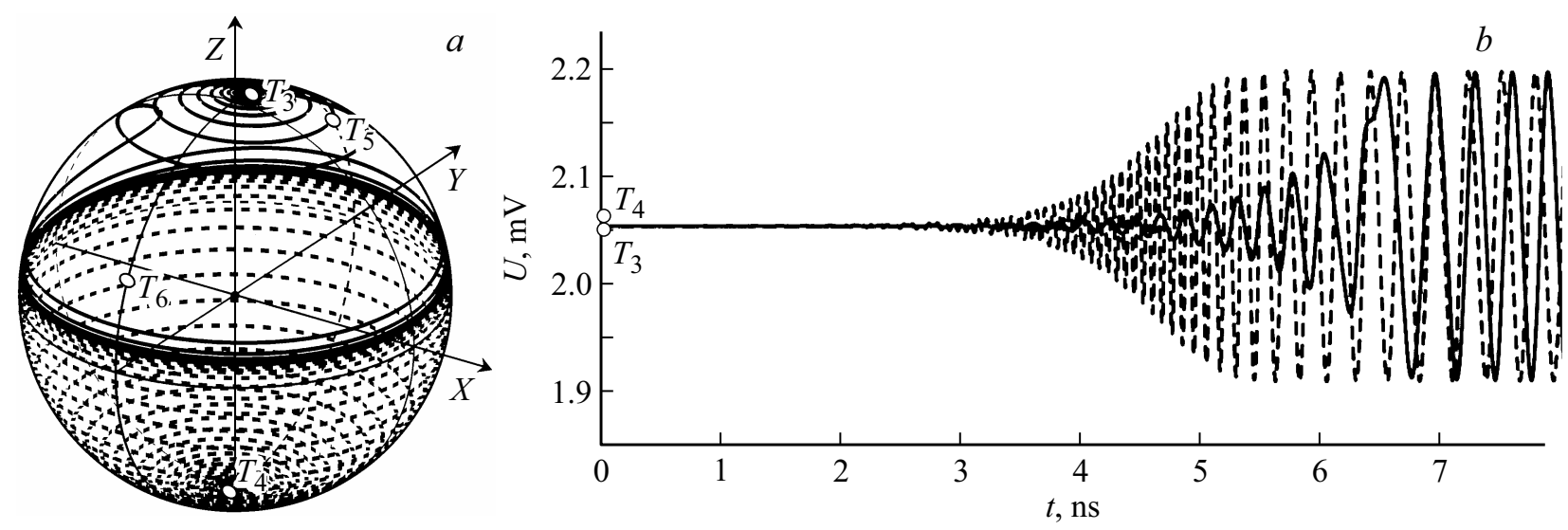

Рис. 6. Устойчивая прецессия вектора намагниченности свободного слоя спинового вентиля на основе железа в магнитном поле параллельном оси $O Z(a)$; вольт-секундные характеристики при тех же параметрах $(b)$.

время, если поле направлено вдоль оси $O X$, возникают два предельных цикла, симметричных относительно этой оси, при этом тип зависимости $U(t)$ близок к пилообразному (рис. $4, b)$.

Из данных табл. 3 следует, что максимальная амплитуда колебаний $U_{\max }$ характерна для спинового вентиля на основе $\mathrm{Fe}_{60} \mathrm{Co}_{20} \mathrm{~B}_{20}$ при $\varphi=\pi / 2, \theta=\pi / 2$. Спиновый вентиль, ферромагнитные слои которого изготовлены из кобальта, имеет максимальную частоту осцилляций при $\varphi=0, \theta=0$. В магнитном поле, направленном вдоль оси $O X$, спиновый вентиль на основе $\mathrm{Co}_{80} \mathrm{Gd}_{20}$ имеет наименьшее энергопотребление, однако амплитуда осцилляций, в этом случае, очень мала.

\section{3. Переключение вектора намагниченности с двумя вероятными исходами}

На рис. 7, $a$ представлена нелинейная модель бинарного стохастического нейрона [25]. В этой модели можно выделить несколько основных элементов:

- набор синаптических связей, каждая из которых характеризуется своим весом $W T$;

- сумматор, который складывает входные сигналы $I$, взвешенные относительно соответствующих синапсов;

- вероятностная функция активации $f\left(I_{\Sigma}\right)$, определяющая конечное состояние нейрона $(+1$ или $1-1)$.

Рис. 7, $b$ демонстрирует модель p-bit, предложенную в данной работе. Малые входные напряжения (менее

Таблица 3. Диапазоны плотности тока $J$ и магнитного поля $H$, при которых наблюдаются предельные циклы, а также частоты $v$ и амплитуды колебаний выходного сигнала $U_{\max }$

\begin{tabular}{|c|c|c|c|c|c|c|}
\hline Материал & $\varphi$ & $\theta$ & $H, \mathrm{MA} / \mathrm{m}$ & $J, \mathrm{MA} / \mathrm{cm}^{2}$ & $v, \mathrm{GHz}$ & $U_{\max }, \mu \mathrm{V}$ \\
\hline Co & $\begin{array}{c}0 \\
\pi / 2 \\
0\end{array}$ & $\begin{array}{c}\pi / 2 \\
\pi / 2 \\
0\end{array}$ & $\begin{array}{l}0.42-0.98 \\
0.56-2.80 \\
1.07-2.80\end{array}$ & $\begin{array}{l}224-1272 \\
234-3516 \\
117-3516\end{array}$ & $\begin{array}{l}2.1-8.0 \\
2.0-17.0 \\
2.4-17.6\end{array}$ & $\begin{array}{c}8.05-90.56 \\
8.81-831.80 \\
10.96-842.97\end{array}$ \\
\hline $\mathrm{Fe}$ & $\begin{array}{c}0 \\
\pi / 2 \\
0\end{array}$ & $\begin{array}{c}\pi / 2 \\
\pi / 2 \\
0\end{array}$ & $\begin{array}{l}0.07-0.79 \\
1.25-1.88 \\
0.50-1.88\end{array}$ & $\begin{array}{l}121-1451 \\
186-4279 \\
186-3721\end{array}$ & $\begin{array}{l}1.8-9.8 \\
1.3-14.3 \\
1.4-9.5\end{array}$ & $\begin{array}{l}14.34-295.57 \\
13.70-2115.80 \\
91.69-1934.40\end{array}$ \\
\hline $\mathrm{Fe}_{70} \mathrm{Co}_{30}$ & $\begin{array}{c}0 \\
\pi / 2 \\
0\end{array}$ & $\begin{array}{c}\pi / 2 \\
\pi / 2 \\
0\end{array}$ & $\begin{array}{l}0.08-1.15 \\
0.14-2.10 \\
0.42-2.10\end{array}$ & $\begin{array}{c}70-835 \\
139-2781 \\
139-2504\end{array}$ & $\begin{array}{c}2.0-8.4 \\
1.4-15.1 \\
1.16-10.4\end{array}$ & $\begin{array}{c}25.25-304.17 \\
15.55-2753.50 \\
128.80-2559.40\end{array}$ \\
\hline $\mathrm{Fe}_{60} \mathrm{Co}_{20} \mathrm{~B}_{20}$ & $\begin{array}{c}0 \\
\pi / 2 \\
0\end{array}$ & $\begin{array}{c}\pi / 2 \\
\pi / 2 \\
0\end{array}$ & $\begin{array}{l}0.47-1.01 \\
0.25-1.87 \\
0.64-1.87\end{array}$ & $\begin{array}{c}232-603 \\
186-2226 \\
93-1948\end{array}$ & $\begin{array}{l}1.7-6.4 \\
1.2-13.2 \\
1.4-7.8\end{array}$ & $\begin{array}{c}72.53-405.42 \\
89.10-4524.20 \\
119.07-4179.90\end{array}$ \\
\hline $\mathrm{Co}_{93} \mathrm{Gd}_{7}$ & $\begin{array}{c}0 \\
\pi / 2 \\
0\end{array}$ & $\begin{array}{c}\pi / 2 \\
\pi / 2 \\
0\end{array}$ & $\begin{array}{r}0.02-0.52 \\
0.07-1.06 \\
1.4-1.06\end{array}$ & $\begin{array}{c}35-778 \\
141-2121 \\
71-1910\end{array}$ & $\begin{array}{l}0.6-4.6 \\
0.3-7.9 \\
0.1-4.8\end{array}$ & $\begin{array}{c}2.09-66.11 \\
2.64-521.63 \\
10.55-412.12\end{array}$ \\
\hline $\mathrm{Co}_{80} \mathrm{Gd}_{20}$ & $\begin{array}{c}0 \\
\pi / 2 \\
0\end{array}$ & $\begin{array}{c}\pi / 2 \\
\pi / 2 \\
0\end{array}$ & $\begin{array}{l}0.02-0.05 \\
0.03-0.12 \\
0.08-0.12\end{array}$ & $\begin{array}{l}2-28 \\
5-51 \\
2-51\end{array}$ & $\begin{array}{c}0.1-0.6 \\
0.02-0.76 \\
0.08-0.46\end{array}$ & $\begin{array}{l}0.01-0.29 \\
0.03-1.53 \\
0.01-1.60\end{array}$ \\
\hline
\end{tabular}


$a$

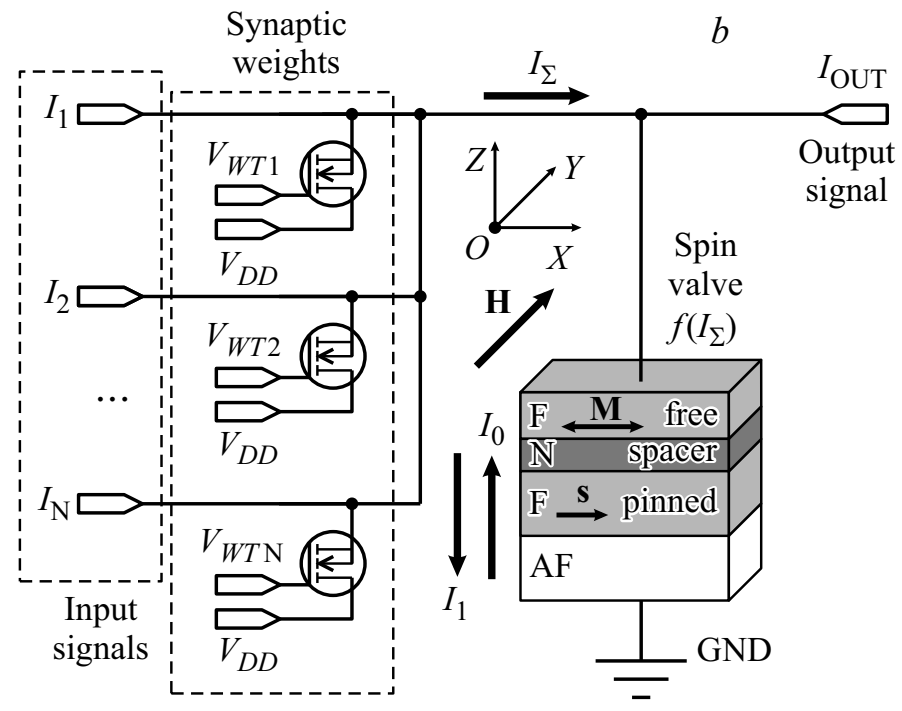

Рис. 7. Стохастический нейрон $(a)$ и предложенная конфигурация p-bit $(b)$.
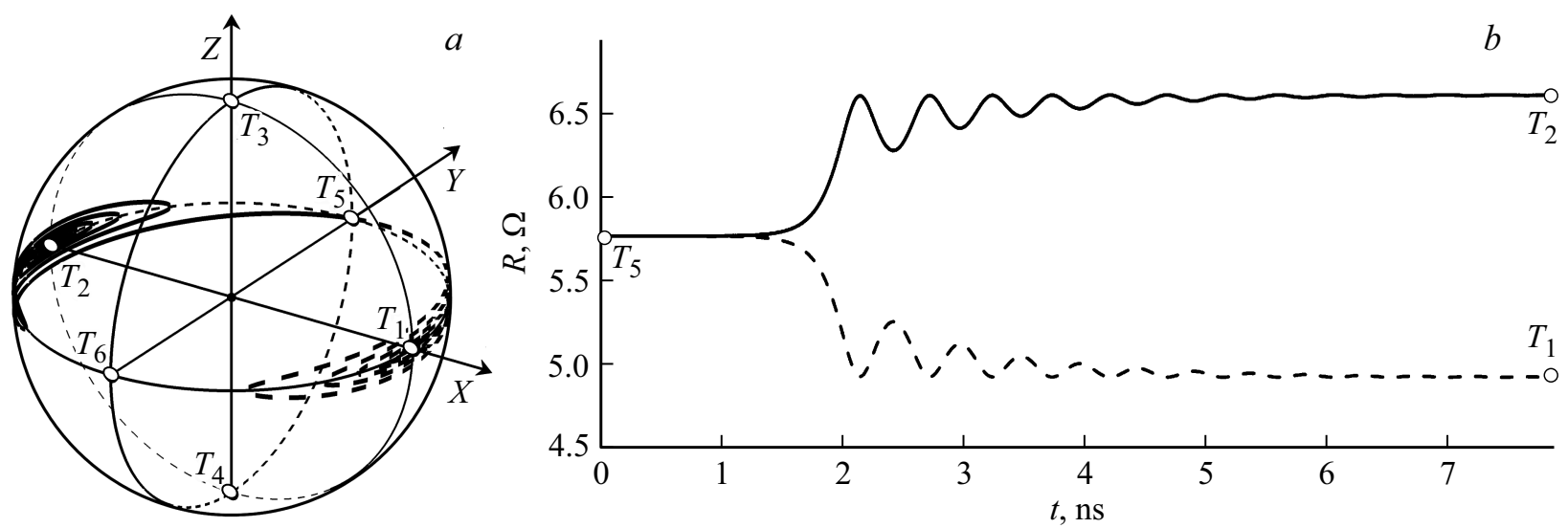

Рис. 8. Переключение вектора М с двумя вероятными исходами для $\mathrm{Fe}_{70} \mathrm{Co}_{30}$ при $H=0$ и $J=0(a)$; временна́я зависимость сопротивления спинового вентиля $R$ при тех же параметрах $(b)$.

$0.01 \mathrm{V)}$ позволяют использовать К-МОП транзисторы как переменные сопротивления для гибкой настройки синаптических весов WT. Спиновый вентиль играет роль элемента вероятностной активационной функции $f\left(I_{\Sigma}\right)$. Основным режимом работы спинового вентиля как элемента PSL является переключение с двумя вероятными исходами (рис. 8). При этом переключении вектор М меняет свое направление с перпендикулярного оси анизотропии $O X$ на параллельное (состояние нейрона „, $\left.+1^{\text {“ }}\right)$ с вероятностью $P_{A P}$, или антипараллельное ей (состояние нейрона „-1“) с вероятностью $P_{P}$.

В работах [9-15] представлена конфигурация бинарного стохастического нейрона p-bit спинового вентиля или MTJ, в которой считывание информации происходит с использованием эффекта GMR, а запись - посредством спинового эффекта Холла. Для реализации записывающего контакта используется шина шириной $1 \mu \mathrm{m}$, что предполагает малую степень интеграции для больших массивов подобных элементов. Модель p-bit, показанная на рисунке рис. $7, b$, лишена этого недостатка, так как считывание и запись информации в ней происходит с помощью эффекта GMR.

Для выведения вектора намагниченности в положение, перпендикулярное оси анизотропии, необходимо приложить магнитное поле, направленное вдоль оси $O Y$ (положение $T_{5}$ ) или $O Z$ (положение $T_{3}$ ). Для этого можно использовать один магнит для всего устройства, что позволит избежать внедрения дополнительных массивных шин в интегральную схему. В этом случае периодически включаемое и выключаемое магнитное поле будет задавать клоковый сигнал для всей интегральной схемы.

В табл. 4 собраны значения минимального магнитного поля $H_{\min 1-5}$, необходимого для переключения из положения $T_{1}$ в положение $T_{5}$, значения аналогичного поля $H_{\min 1-3}$ для положения $T_{3}$, а также соответствующие времена перемагничивания $t_{1-5}$ и $t_{1-3}$. При этом магнитное поле $H_{\min 1-5}$, значительно ниже, 
Таблица 4. Переключательные параметры p-bit на основе различных материалов

\begin{tabular}{l|c|c|c|c|r|r|r}
\hline Материал & $\begin{array}{c}H_{\min 1-5}, \\
\mathrm{MA} / \mathrm{m}\end{array}$ & $\begin{array}{c}t_{\max 1-5}, \\
\mathrm{~ns}\end{array}$ & $\begin{array}{c}H_{\min 1-3}, \\
\mathrm{MA} / \mathrm{m}\end{array}$ & $\begin{array}{c}t_{\max 1-3,}, \\
\mathrm{~ns}\end{array}$ & $\begin{array}{c}J_{\max }{ }^{2} \\
\mathrm{kA} / \mathrm{cm}^{2}\end{array}$ & $\begin{array}{c}t_{\min }, \\
\mathrm{ns}\end{array}$ & $\begin{array}{c}t_{\max }, \\
\mathrm{ns}\end{array}$ \\
\hline $\mathrm{Co}$ & 0.565 & 2.9 & 1.983 & 9.7 & 448.8 & 4.6 & 5.0 \\
$\mathrm{Fe}$ & 0.023 & 0.8 & 1.734 & 98.3 & 472.2 & 10.1 & 12.2 \\
$\mathrm{Fe}_{70} \mathrm{Co}_{30}$ & 0.026 & 4.0 & 1.931 & 113.3 & 403.4 & 5.0 & 6.7 \\
$\mathrm{Fe}_{60} \mathrm{Co}_{20} \mathrm{~B}_{20}$ & 0.218 & 3.1 & 1.778 & 15.7 & 296.8 & 2.9 & 3.4 \\
$\mathrm{Co}_{93} \mathrm{Gd}_{7}$ & 0.005 & 10.4 & 1.170 & 27.2 & 213.8 & 8.9 & 13.3 \\
$\mathrm{Co}_{80} \mathrm{Gd}_{20}$ & 0.028 & 133.9 & 0.107 & 265.8 & 7.8 & 84.5 & 93.2
\end{tabular}

чем $H_{\min 1-3}$. В табл. 4 приведены значения величины плотности тока $J_{\max }$ необходимой для переключения p-bit из положения $T_{5}$ в положение $T_{2}$ с вероятностью $P_{A P}=100 \%$, а также время переключения $t_{\min }$ при таком токе. Для переключения в положение $T_{1}$ с вероятностью $P_{P}=100 \%$ необходимо пропустить противоположный по направлению ток $J_{\max }$ такой же величины. Время переключения при нулевом токе $t_{\max }\left(P_{P}=P_{A P}=50 \%\right)$ также представлено в табл. 4.

Среди рассмотренных материалов наименьшим значением $H_{\min 1-5}$ обладает спиновый вентиль, изготовленный из сплава $\mathrm{Co}_{93} \mathrm{Gd}_{7}$, в то время как наименьший ток $J_{\max }$ соответствует $\mathrm{Co}_{80} \mathrm{Gd}_{20}$. Наибольшее быстродействие показал p-bit на основе $\mathrm{Fe}_{60} \mathrm{Co}_{20} \mathrm{~B}_{20}$.

\section{4. Заключение}

В работе численно рассчитаны зависимости времени переключения спинового вентиля из параллельного состояния в антипараллельное от величины спинполяризованного тока $t_{1-2}(J)$ и времени обратного переключения от величины магнитного поля, параллельного оси анизотропии $t_{2-1}(H)$. Установлено, что наиболее подходящим материалом из рассмотренных для реализации MRAM, является $\mathrm{Co}_{80} \mathrm{Gd}_{20}$, так как спиновый вентиль на основе этого материала имеет наименьший критический ток переключения $J_{\min }$ и самую высокую скорость переключения спин-поляризованным током. Минимальная сила тока, достаточная для переключения ячейки MRAM, в этом случае составляет всего $2.4 \mu \mathrm{A}$, что в 3 раза меньше, чем для материала, использованного корпорацией IBM в работе [4]. Обнаружено, что оптимальным материалом для изготовления считывающих головок HMDD является $\mathrm{Fe}_{60} \mathrm{Co}_{20} \mathrm{~B}_{20}$, так как он имеет лучшее соотношения коэффициента GMR минимального поля переключения $H_{\min }$ и наименьшее время переключения магнитным полем. Полученные данные согласуются с результатами, изложенными в работе [17].

Рассчитаны диапазоны токов и магнитных полей, при которых наблюдается устойчивая прецессия вектора намагниченности свободного слоя спинового вентиля. При этом рассматривались три ортогональных направления магнитного поля. Показано, что для получения сигнала, близкого к пилообразному, необходимо использовать магнитное поле, параллельное оси анизотропии. В то же время, гармоническому сигналу соответствуют направления, перпендикулярные оси анизотропии. Установлено, что сплав $\mathrm{Fe}_{60} \mathrm{Co}_{20} \mathrm{~B}_{20}$ является наиболее подходящим из рассмотренных для изготовления STNO, так как спиновый вентиль на его основе обладает максимальной амплитудой выходного сигнала в магнитном поле, параллельном оси $O Y$. При этом диапазоны токов и полей в данном случае в 1.5 раза ниже, чем для спинового вентиля на основе кобальта, имеющего максимальную частоту выходного сигнала.

Предложена принципиально новая модель бинарного стохастического нейрона. Обнаружено, что для этой модели p-bit на основе $\mathrm{Co}_{80} \mathrm{Gd}_{20}$ имеет наименьшее энергопотребление, а нейрон на основе $\mathrm{Fe}_{60} \mathrm{Co}_{20} \mathrm{~B}_{20}$ наибольшее быстродействие. Однако по совокупности этих параметров оптимальным материалом для изготовления p-bit является $\mathrm{Co}_{93} \mathrm{Gd}_{7}$. При этом величина силы тока, достаточная для перехода p-bit в состояние „+1“ с вероятностью $100 \%$, в этом случае составляет всего $0.26 \mu \mathrm{A}$, в то время как интеграции такого элемента 50 раз выше, чем для модели p-bit, представленной в работах [9-15].

Приведенные расчетные данные имеют общий рекомендательный характер и служат иллюстрацией использования теоретической модели спинового вентиля.

\section{Благодарности}

Автор благодарит Н.В. Островскую за полезное обсуждение задачи и доброжелательное прочтение рукописи, а также А.С. Михмеля за ценную консультацию по вопросам технологии СБИС.

\section{Финансирование работы}

Работа выполнена в рамках государственного задания „Исследование и разработка высокопроизводительных вычислительных систем и элементной базы для них“ шифр „Вега-Ст-2021“, внутренний номер 0067-20190005, № 2P AAAA-A19-119043090023-6.

\section{Конфликт интересов}

Автор заявляет, что у него нет конфликта интересов. 


\section{Список литературы}

[1] J.C. Slonczewski. J. Magn. Magn. Mater. 159, L1 (1996).

[2] N.V. Ostrovskaya, V.A. Skidanov, Iu.A. Iusipova. Solid State Phenomena 233-234, 431 (2015).

[3] C. Chappert, A. Fert, F. Nguyen Van Dau. Nature Mater. 6, 813. (2007).

[4] J.J. Nowak, R.P. Robertazzi, J.Z. Sun, G. Hu, J.H. Park, J.H. Lee, A.J. Annunziata, G.P. Lauer, C. Kothandaraman, E.J. O’Sullivan, P.L. Trouilloud, Y. Kim, D.C. Worledge. IEEE Magn. Lett. 7, 3102604 (2016).

[5] Yimin Guo. US Patent No US0151614A1 (2008).

[6] Ch.-Y. You. J. Magn. 14, 4, 168 (2009).

[7] Z. Zeng, G. Finocchio, H. Jiang. Nanoscale 5, 6, 2219 (2013).

[8] N. Locatelli, R. Lebrun, V.V. Naletov, A. Hamadeh, G. De Loubens, O. Klein, J. Grollier, V. Cros. IEEE Trans. Magn. 51, 4300206 (2015).

[9] S. Datta, S. Salahuddin, B. Behin-Aein. Appl. Phys. Lett. 101, 252411 (2012).

[10] B. Behin-Aein, V. Diep, S. Datta. Sci. Rep. 6, 1 (2016).

[11] K.Y. Camsari, R. Faria, B.M. Sutton, S. Datta. Phys. Rev. X 7, 3, 031014 (2017).

[12] B. Sutton, K.Y. Camsari, B. Behin-Aein, S. Datta. Sci. Rep. 7, 1 (2017).

[13] Y. Shim, A. Jaiswal, K. Roy. J. Appl. Phys. 121, 193902 (2017)

[14] R. Faria, K.Y. Camsari, S. Datta. IEEE Magn. Lett. 8, 2685358 (2017)

[15] R. Zand, K.Y. Camsari, S.D. Pyle, I. Ahmed, C.H. Kim, R.F. DeMara. In: Proc. of the 2018 on Great Lakes Symposium on VLSI.ACM (2018). P. 15-20.

[16] E.E. Shalygina, A.V. Makarov, A.M. Kharlamova, G.V. Kurlyandskaya, A.V. Svalov. Reviews and Short Notes to Nanomeeting-2017: Physics, chemistry and application of nanostructures (2017). P. 89-92.

[17] Iu.A. Iusipova. Semicond. 52, 15, 1982 (2018).

[18] Iu.A. Iusipova. Semicond. 53, 15, 2029 (2019).

[19] Ю.А. Юсипова. Изв. вузов. Электроника 24, 5, 489 (2019).

[20] R.R. Katti. Proc. IEEE 91, 687 (2003).

[21] E.Y. Tsymbal, I. Žutić. Handbook of Spin Transport and Magnetism. CRC Press (2012). 777 p.

[22] А.П. Бабичев, Н.А. Бабушкина, А.М. Братковский. Физические величины. Справочник / Под ред. И.С. Григорьева, Е.3. Мейлихова. Энергоатомиздат, М., (1991). 1232 с.

[23] В.В. Пасынков, В.С. Сорокин. Материалы электронной техники. Учебное пособие для вузов по специальности электронной техники. 3 изд. Изд-во „Лань“, СПб. (2001). $368 \mathrm{c}$.

[24] S.X. Huang, T.Y. Chen, C.L. Chien. Appl. Phys. Lett. 92, 242509 (2008).

[25] S. Haykin. Neural networks and learning machines. 3rd ed. Prentice Hall, New Jersey (2008). 977 p.

Редактор Ю.Э. Китаев 